\title{
Misdiagnosis of narcolepsy
}

\author{
Laura Dunne $^{1} \cdot$ Pallavi Patel $^{2} \cdot$ Emily L Maschauer $^{1} \cdot$ Ian Morrison $^{1,2} \cdot$ Renata L Riha $^{1}$
}

Received: 4 November 2015 /Revised: 16 May 2016 / Accepted: 23 May 2016/Published online: 23 June 2016

(C) The Author(s) 2016. This article is published with open access at Springerlink.com

\begin{abstract}
Background Narcolepsy is a chronic primary sleep disorder, characterized by excessive daytime sleepiness and sleep dysfunction with or without cataplexy. Narcolepsy is uncommon, with a low prevalence rate which makes it difficult to diagnose definitively without a complex series of tests and a detailed history. The aim of this study was to review patients referred to a tertiary sleep centre who had been labelled with a diagnosis of narcolepsy prior to referral in order to assess if the diagnosis was accurate, and if not, to determine the cause of diagnostic misattribution.

Methods All patients seen at a sleep centre from 2007-2013 ( $n=551)$ who underwent detailed objective testing including an MSLT PSG, as well as wearing an actigraphy watch and completing a sleep diary for 2 weeks, were assessed for a prereferral and final diagnosis of narcolepsy.

Results Of the 41 directly referred patients with a diagnostic label of narcolepsy, 19 (46\%) were subsequently confirmed to have narcolepsy on objective testing and assessment by a sleep physician using ICSD-2 criteria.

Conclusions The diagnosis of narcolepsy was incorrectly attributed to almost $50 \%$ of patients labelled with a diagnosis of narcolepsy who were referred for further opinion by a variety of specialists and generalists. Accurate diagnosis of narcolepsy is critical for many reasons, such as
\end{abstract}

Renata L Riha

rlriha@hotmail.com

1 Sleep Research Unit, Department of Sleep Medicine, University of Edinburgh, Royal Infirmary of Edinburgh, 51 Little France Crescent, Edinburgh EH16 4SA, UK

2 Department of Neurology, University of Dundee, Ninewells Hospital, Dundee DD1 9SY, UK the impact it has on quality of life, driving, employment, insurance and pregnancy in women as well as medication management.

Keywords Narcolepsy $\cdot$ Diagnosis $\cdot$ Misdiagnosis ·

Cataplexy $\cdot$ MSLT

\section{Introduction}

Narcolepsy is a chronic primary sleep disorder, characterized by excessive daytime sleepiness (EDS) with symptoms of rapid eye movement (REM) and sleep dysfunction (i.e. sleep paralysis, hypnagogic hallucinations), with or without cataplexy (muscle weakness). Narcolepsy is uncommon and the estimated prevalence ranges from 0.01 to $0.05 \%$ worldwide. [1] The low prevalence means a definitive diagnosis of narcolepsy can be complex and should incorporate a detailed presenting history, assessment of sleep-wake cycles, assessment of sleep deprivation, subjective testing (sleep diary) and objective testing (polysomnography (PSG), actigraphy, multiple sleep latency testing (MSLT) including drug-testing of urine, human leukocyte antigen (HLA) typing and lumbar puncture to measure CSF hypocretin-1 (orexin) levels) [2].

Even with the use of the International Classification of Sleep Disorders-2 (ICSD-2) [9] and the International Classification of Sleep Disorders-3 (ICSD-3) [10] (since 2014), uncertainty with diagnosis may persist with the most experienced clinicians, given that the symptoms of narcolepsy can often be mimicked by other sleep disorders (e.g. sleep disordered breathing) [11] or overlap with psychiatric symptomology. [8] Patients have been misdiagnosed with narcolepsy on the basis of clinical judgement alone, resulting in inappropriate treatment. [3, 4] The aim of this study was to review patients who had been labelled with a diagnosis of 
narcolepsy by a variety of specialists and generalists prior to referral to a tertiary sleep centre for a second opinion and/or continued management, as well as to determine the basis for any diagnostic misattribution.

\section{Methods}

A database comprising all patients seen at a tertiary referral sleep centre undergoing MSLT between October 2007 and July $2013(n=551)$ was cross-examined. 'Diagnosis of narcolepsy' on the referral letter was defined as patients given a diagnosis of 'narcolepsy', patients labelled with 'probable narcolepsy' (with/without treatment) and patients deemed as 'possibly' having narcolepsy provided they had received treatment with stimulant medication prior to referral. All patient referrals came from regional centres that did not have expertise in non-respiratory sleep medicine. Most patients had received little to no assessment or testing for narcolepsy before presenting at the sleep clinic (very few had a limited study or PSG, but none had an MSLT). The ICSD-2 [9] was used to determine criteria for the narcolepsy and cataplexy diagnosis. All patients were subsequently reviewed by one of two sleep specialists in the clinic. Given the frequency of comorbidity of other sleep disorders in the narcolepsy population (e.g. obstructive sleep apnoea), all patients underwent an MSLT and PSG and wore an actigraphy watch and kept a sleep diary for 2 weeks prior to in-lab testing. All patients underwent further assessment in the sleep clinic to ensure that symptoms were more likely as a result of narcolepsy and not due to other sleep disorders.

Sole reliance for diagnosis was not placed on the MSLT. The MSLT should not be used as a diagnostic tool in isolation, [15] due to its limited test-retest reliability, sometimes yielding misleading or false positive or negative results, [12] and its inadequate exclusion of other sleep disorders (e.g. OSAHS). [3] Arand et al. [15] recommend improving the reliability of the MSLT by combining it with the results from a PSG and obtaining a suitable history of the patient. The sleep specialists reviewed the referrals in addition to obtaining detailed history directly from the patient and acquiring information from objective test results from actigraphy over 2 weeks, PSG followed by MSLT, a urine drug screen, as well as a subjective 2week sleep diary. Difficult cases were discussed at multidisciplinary meetings and diagnosis was achieved by consensus and further review. Investigation and scoring of sleep data were conducted in a blinded manner; technicians who scored the data were not involved with history taking nor allowed to review the patient files to prevent any bias in scoring. Specific ethical approval was not considered necessary, as the Ethics Advisory Committee in Edinburgh does not require approval for case series.
Data were gathered on presenting history, medication, referral/diagnosis, objective investigations, co-morbidities and anthropometrics. Analysis, using standard statistical techniques, was undertaken with SPSS (IBM, v.19). Significance was taken at $p \leq 0.05$, and all tests were two-tailed.

\section{Results}

Of the 41 patients referred with a 'diagnosis of narcolepsy' over the 6-year period, only 19 (46\%) were confirmed to have narcolepsy after further assessment and objective testing. The source of the initial diagnosis in the majority $(n=18)$ was a general practitioner; in the remainder, diagnoses had been made by a neurologist $(n=8)$, a regional sleep clinic $(n=9)$, or respiratory physician $(n=6)$.

Females were significantly more likely to be diagnosed with narcolepsy than males ( 15 vs. $7 ; p=0.002$ ).

A history of cataplexy was present in $84 \%$ of the confirmed narcoleptic patients and $27 \%$ of the pre-reported non-narcoleptics $(p<0.001)$. EDS was measured subjectively by the Epworth sleepiness scale, [5] and the results were equivalent between groups $(15.15 \pm 5.43$ in narcoleptics vs. $15.50 \pm 4.53$ in non-narcoleptics, $p=0.85$ ).

The median sleep onset latency on overnight PSG was lower in patients with confirmed narcolepsy than for patients without confirmed narcolepsy $(6.5 \pm 7.86, p=0.005$ vs. $21.5 \pm 34.63, p<0.0001)$, as was mean sleep latency on $\operatorname{MSLT}(3.65 \pm 2.95$ vs. $10.20 \pm 4.86, p<0.0001)$. All confirmed narcoleptics experienced at least two SOREMPs on the MSLT. Six non-narcoleptics were able to achieve REM sleep on the MLST. Mean REM latency was significantly lower in the confirmed narcoleptics vs. non-narcoleptics (2.7 vs. $7.8 \mathrm{~min}, p=0.007)$. The number of sleep onset REMperiods was greater in patients with confirmed narcolepsy compared to those without $(3.1 \pm 1.1$ vs $1.6 \pm 0.8$, $p=0.004)$. DQB $1 * 0602$ alleles were found in $90 \%$ of the confirmed narcolepsy patients, in $40 \%$ of the non-narcoleptic patients $(p=0.037)$ and in the three $(16 \%)$ patients who had narcolepsy without cataplexy. Despite the fact that $90-95 \%$ of the narcolepsy population will have a positive HLA for narcolepsy, [13] we used the HLA markers as an additive factor, not the only component, to support the diagnosis of narcolepsy. Three non-narcoleptic patients who had CSF-hypocretin-1 measured had entirely normal levels (>110 pg/ml).

Only three patients without narcolepsy had no discernible explanation for their symptoms. Diagnoses in the remaining 19 were obstructive sleep apnoea syndrome $(n=10)$, depression/anxiety $(n=3)$, sleep deprivation $(n=3)$, irregular sleep/wake cycles $(n=2)$ and parasomnia $(n=1)$ (Table 1). Thus, non-narcoleptics and patients with EDS differed from true narcoleptics determined by the assessment tests described above. 
Table 1 Demographic and diagnosis information at referral for 41 patients referred with a 'diagnosis of narcolepsy'

\begin{tabular}{|c|c|c|c|c|c|c|c|c|c|}
\hline Case & Age & $\begin{array}{l}\text { Prior } \\
\text { diagnosis }\end{array}$ & Medication & PSG results & MSLT results & $\begin{array}{l}\text { Drug } \\
\text { screen }^{\mathrm{a}}\end{array}$ & HLA type & $\begin{array}{l}\text { Features } \\
\text { of } \\
\text { cataplexy }\end{array}$ & $\begin{array}{l}\text { Final } \\
\text { diagnosis }\end{array}$ \\
\hline 1 & 28 & $\begin{array}{l}\text { Probable } \\
\mathrm{Nx}\end{array}$ & None & $\begin{array}{l}\text { SE: } 87.6 \% \\
\text { SOLmin: } 3.4 \\
\text { REML: } 0 \text { min } \\
\text { AHI: } 52.9 \\
\quad \text { events/h }\end{array}$ & $\begin{array}{l}\text { MSL: } 2.0 \text { min } \\
\text { MREML: } 0.3 \\
\text { No. naps with } \\
\text { REM: } 4\end{array}$ & Negative & $\begin{array}{l}\text { Blood could } \\
\text { not be } \\
\text { obtained or } \\
\text { patient } \\
\text { refused }\end{array}$ & Present & True Nx \\
\hline 2 & 26 & $\begin{array}{l}\text { Probable } \\
\text { Nx }\end{array}$ & None & $\begin{array}{l}\text { SE: } 84.6 \% \\
\text { SOLmin: } 2.5 \\
\text { REML: } 0.5 \mathrm{~min} \\
\text { AHI: } 10.1 \\
\quad \text { events } / \mathrm{h}\end{array}$ & $\begin{array}{l}\text { MSL: } 1.9 \text { min } \\
\text { MREML: } 2.6 \\
\text { No. naps with } \\
\text { REM: } 4\end{array}$ & Negative & DQB1*06:02 & Present & True Nx \\
\hline 3 & 56 & $\mathrm{Nx}$ & $\begin{array}{l}\text { Dipyridamole, ezetimibe, } \\
\text { aspirin, perindopril } \\
\text { erbumine, bendroflumet- } \\
\text { hiazide, co-codamol }\end{array}$ & $\begin{array}{l}\text { SE: } 67.3 \% \\
\text { SOLmin: } 26.0 \\
\text { REML: } 52.0 \mathrm{~min} \\
\text { AHI: } 34.6 \\
\quad \text { events/h }\end{array}$ & $\begin{array}{l}\text { MSL: } 8.2 \text { min } \\
\text { MREML: } \\
11.0 \\
\text { No. naps with } \\
\text { REM: } 1\end{array}$ & Negative & Negative & Absent & OSAHS \\
\hline 4 & 55 & $\mathrm{Nx}$ & $\begin{array}{l}\text { Aspirin, bisoprolol, clenil } \\
\text { modulite, co-dydramol, } \\
\text { furosemide, nitroglycerin, } \\
\text { nicorandil, ramipril, } \\
\text { salbutamol, simvastatin }\end{array}$ & $\begin{array}{l}\text { SE: } 51.5 \% \\
\text { SOLmin: } 161.0 \\
\text { REML: } 95.0 \mathrm{~min} \\
\text { AHI: } 17.8 \\
\quad \text { events/h }\end{array}$ & $\begin{array}{l}\text { MSL: } \\
18.0 \text { min } \\
\text { MREML: } 0 \\
\text { No. naps with } \\
\text { REM: } 0\end{array}$ & Negative & Negative & Absent & $\begin{array}{l}\text { Depression/ } \\
\text { anxiety }\end{array}$ \\
\hline 5 & 65 & $\begin{array}{l}\text { Probable } \\
\text { Nx }\end{array}$ & None & $\begin{array}{l}\text { SE: } 73.4 \% \\
\text { SOLmin: } 38.0 \\
\text { REML: } \\
45.5 \text { min AHI: } \\
13.6 \text { events } / \mathrm{h}\end{array}$ & $\begin{array}{l}\text { MSL: } \\
15.5 \text { min } \\
\text { MREML: } \\
\text { 0 No. naps } \\
\text { with REM: } \\
0\end{array}$ & Negative & Negative & Absent & OSAHS \\
\hline 6 & 37 & $\mathrm{Nx}$ & None & $\begin{array}{l}\text { SE: } 88.2 \% \\
\text { SOLmin: } 9.0 \\
\text { REML: } 59.0 \mathrm{~min} \\
\text { AHI: } 8.7 \text { events/h }\end{array}$ & $\begin{array}{l}\text { MSL: } \\
11.6 \text { min } \\
\text { MREML: } 0 \\
\text { No. naps with } \\
\text { REM: } 0\end{array}$ & $\begin{array}{l}\text { Urine could } \\
\text { not be } \\
\text { obtained } \\
\text { or patient } \\
\text { refused }\end{array}$ & $\begin{array}{l}\text { DQB1*0603/ } \\
\text { 08/14, } \\
\text { HLA- } \\
\text { DQ- } \\
\text { A1*0103 }\end{array}$ & Present & $\begin{array}{l}\text { Depression/ } \\
\text { anxiety }\end{array}$ \\
\hline 7 & 31 & $\mathrm{Nx}$ & None & $\begin{array}{l}\text { SE: } 85.1 \% \\
\text { SOLmin: } 6.0 \\
\text { REML: } 1.0 \mathrm{~min} \\
\text { AHI: } 10.7 \\
\quad \text { events } / \mathrm{h}\end{array}$ & $\begin{array}{l}\text { MSL: } 4.4 \text { min } \\
\text { MREML: } 1.0 \\
\text { No. naps with } \\
\text { REM: } 3\end{array}$ & Negative & DQB1*06:02 & Absent & True Nx \\
\hline 8 & 31 & $\begin{array}{l}\text { Probable } \\
\mathrm{Nx}\end{array}$ & Lansoprazole, thyroxine & $\begin{array}{l}\text { SE: } 83.0 \% \\
\text { SOLmin: } 26.5 \\
\text { REML: } \\
95.5 \text { min AHI: } \\
23.4 \text { events/h }\end{array}$ & $\begin{array}{l}\text { MSL: } 3.9 \text { min } \\
\text { MREML: } \\
13.3 \\
\text { No. naps with } \\
\text { REM: } 2\end{array}$ & Negative & DQB $1 * 0602$ & Present & $\begin{array}{l}\text { Sleep } \\
\text { deprivation }\end{array}$ \\
\hline 9 & 23 & $\begin{array}{l}\text { Probable } \\
\text { Nx }\end{array}$ & $\begin{array}{l}\text { Salbutamol, oral contraceptive, } \\
\text { betamethasone cream, } \\
\text { beclomethasonedipropionate } \\
\text { inhaler, }\end{array}$ & $\begin{array}{l}\text { SE: } 93.0 \% \\
\text { SOLmin: } 6.0 \\
\text { REML: } 57.0 \mathrm{~min} \\
\text { AHI: } 8.2 \text { events/h }\end{array}$ & $\begin{array}{l}\text { MSL: } \\
11.3 \text { min } \\
\text { MREML: } 0 \\
\text { No. naps with } \\
\text { REM: } 0\end{array}$ & Negative & $\begin{array}{l}\text { Blood could } \\
\text { not be } \\
\text { obtained or } \\
\text { patient } \\
\text { refused }\end{array}$ & Absent & True Nx \\
\hline 10 & 65 & $\mathrm{Nx}$ & $\begin{array}{l}\text { Nitroglycerin, co-codamol, } \\
\text { frusemide, telmisartan, } \\
\text { rosuvastatin, cordarone, } \\
\text { warfarin }\end{array}$ & $\begin{array}{l}\text { SE: } 52.1 \% \\
\text { SOLmin: } 1.5 \\
\text { REML: } \\
223.5 \text { min } \\
\text { AHI: } 25.6 \\
\text { events/h }\end{array}$ & $\begin{array}{l}\text { MSL: } 8.0 \text { min } \\
\text { MREML: } 0 \\
\text { No. naps with } \\
\text { REM: } 0\end{array}$ & Negative & $\begin{array}{l}\text { Positive but } \\
\text { type not } \\
\text { recorded }\end{array}$ & Absent & OSAHS \\
\hline 11 & 50 & $\begin{array}{l}\text { Probable } \\
\text { Nx }\end{array}$ & Clonidine & $\begin{array}{l}\text { SE: } 68.2 \% \\
\text { SOLmin: } 70.0 \\
\text { REML: } \\
322.0 \mathrm{~min} \\
\text { AHI: } 10.3 \\
\text { events/h }\end{array}$ & $\begin{array}{l}\text { MSL: } \\
14.0 \text { min } \\
\text { MREML: } 0 \\
\text { No. naps with } \\
\text { REM: } 0\end{array}$ & Positive & $\begin{array}{l}\text { Blood could } \\
\text { not be } \\
\text { obtained or } \\
\text { patient } \\
\text { refused }\end{array}$ & Present & $\begin{array}{l}\text { Irregular } \\
\text { sleep }\end{array}$ \\
\hline 12 & 51 & $\mathrm{Nx}$ & None & $\begin{array}{l}\text { SE: } 85.3 \% \\
\text { SOLmin: } 6.5\end{array}$ & $\begin{array}{l}\text { MSL: } 1.8 \mathrm{~min} \\
\text { MREML: } 6.3\end{array}$ & Negative & & Present & True Nx \\
\hline
\end{tabular}


Table 1 (continued)

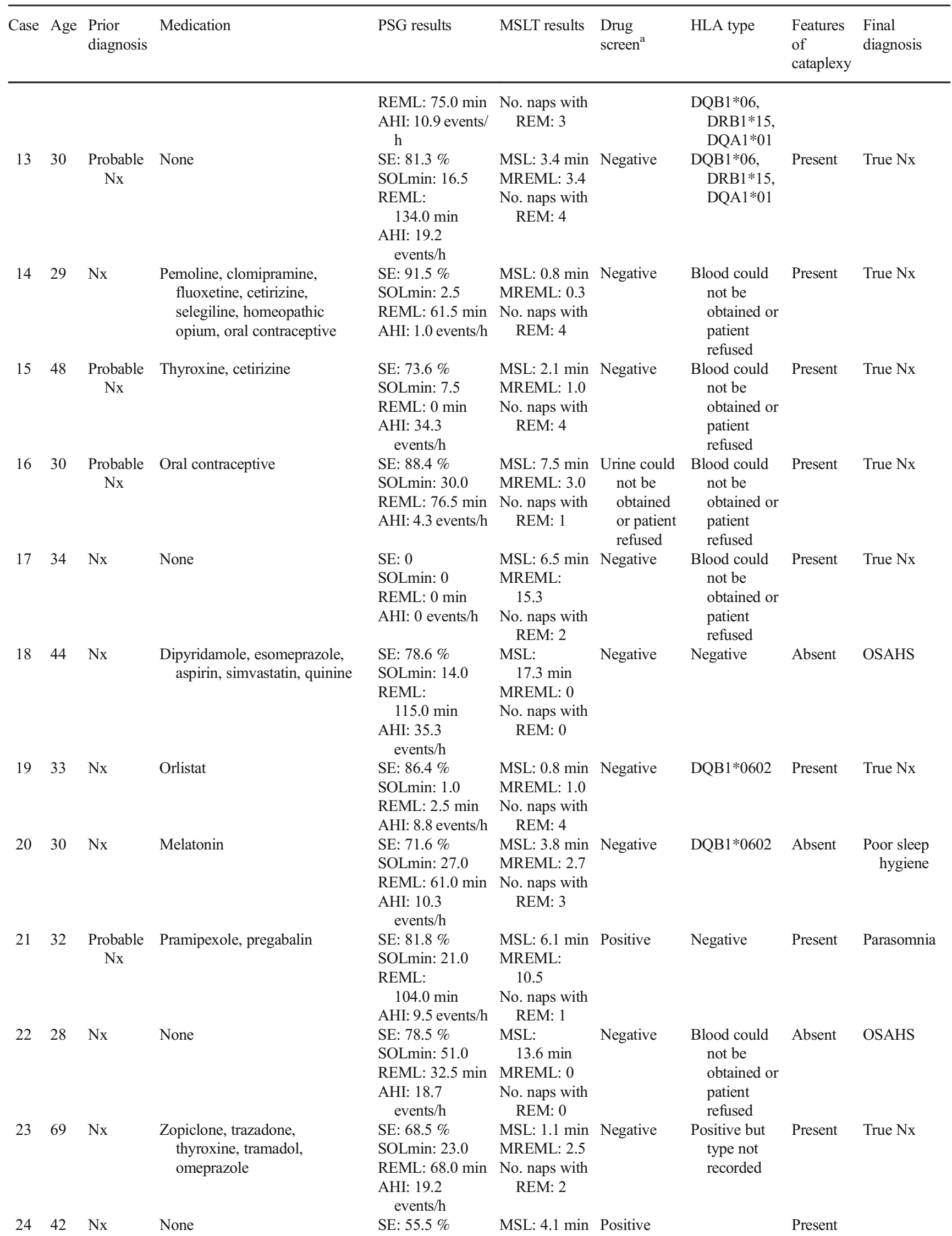


Table 1 (continued)

\begin{tabular}{|c|c|c|c|c|c|c|c|c|c|}
\hline Case & Age & $\begin{array}{l}\text { Prior } \\
\text { diagnosis }\end{array}$ & Medication & PSG results & MSLT results & $\begin{array}{l}\text { Drug } \\
\text { screen }^{\mathrm{a}}\end{array}$ & HLA type & $\begin{array}{l}\text { Features } \\
\text { of } \\
\text { cataplexy }\end{array}$ & $\begin{array}{l}\text { Final } \\
\text { diagnosis }\end{array}$ \\
\hline & & & & $\begin{array}{l}\text { SOLmin: } 7.5 \\
\text { REML: } 89.0 \mathrm{~min} \\
\text { AHI: } 30.5 \\
\quad \text { events/h }\end{array}$ & $\begin{array}{l}\text { MREML: } 1.3 \\
\text { No. naps with } \\
\text { REM: } 2\end{array}$ & & $\begin{array}{c}\text { DQB1*0602/ } \\
14 / 15 / 16 / \\
19 / 20 / 23 / \\
24 / 33\end{array}$ & & $\begin{array}{l}\text { Depression/ } \\
\text { anxiety }\end{array}$ \\
\hline 25 & 27 & $\begin{array}{l}\text { Probable } \\
\mathrm{Nx}\end{array}$ & None & $\begin{array}{l}\text { SE: } 84.0 \% \\
\text { SOLmin: } 11.5 \\
\text { REML: } 0.5 \mathrm{~min} \\
\text { AHI: } 3.7 \text { events/h }\end{array}$ & $\begin{array}{l}\text { MSL: } 2.5 \text { min } \\
\text { MREML: } 3.6 \\
\text { No. naps with } \\
\text { REM: } 4\end{array}$ & Negative & DQB1*0602 & Present & True Nx \\
\hline 26 & 31 & $\begin{array}{l}\text { Probable } \\
\mathrm{Nx}\end{array}$ & None & $\begin{array}{l}\text { SE: } 84.6 \% \\
\text { SOLmin: } 12.5 \\
\text { REML: } \\
\quad 192.5 \text { min } \\
\text { AHI: } 6.2 \text { events/h }\end{array}$ & $\begin{array}{l}\text { MSL: } 7.0 \text { min } \\
\text { MREML: } 0 \\
\text { No. naps with } \\
\text { REM: } 0\end{array}$ & Positive & DQB1*0602 & Absent & $\begin{array}{l}\text { Sleep } \\
\text { deprivation }\end{array}$ \\
\hline 27 & 32 & $\mathrm{Nx}$ & Mazindol & $\begin{array}{l}\text { SE: } 84.8 \% \\
\text { SOLmin: } 7.0 \\
\text { REML: } 55.5 \mathrm{~min} \\
\text { AHI: } 14.4 \\
\quad \text { events/h }\end{array}$ & $\begin{array}{l}\text { MSL: } 2.4 \text { min } \\
\text { MREML: } 1.3 \\
\text { No. naps with } \\
\text { REM: } 4\end{array}$ & Positive & $\begin{array}{l}\text { Blood could } \\
\text { not be } \\
\text { obtained or } \\
\text { patient } \\
\text { refused }\end{array}$ & Present & True Nx \\
\hline 28 & 42 & $\begin{array}{l}\text { Probable } \\
\mathrm{Nx}\end{array}$ & Omeprazole & $\begin{array}{l}\text { SE: } 94.2 \% \\
\text { SOLmin: } 4.5 \\
\text { REML: } \\
59.0 \text { min AHI: } \\
26.1 \text { events/h }\end{array}$ & $\begin{array}{l}\text { MSL: } 5.1 \text { min } \\
\text { MREML: } \\
\text { 5.5 No. } \\
\text { naps with } \\
\text { REM: } 1\end{array}$ & Positive & Negative & Absent & OSAHS \\
\hline 29 & 30 & $\mathrm{Nx}$ & Amitriptyline, modafinil & $\begin{array}{l}\text { SE: } 86.3 \% \\
\text { SOLmin: } 34.5 \\
\text { REML: } 54.0 \mathrm{~min} \\
\text { AHI: } 6.9 \text { events/h }\end{array}$ & $\begin{array}{l}\text { MSL: } 7.9 \text { min } \\
\text { MREML: } 0 \\
\text { No. naps with } \\
\text { REM: } 0\end{array}$ & $\begin{array}{l}\text { Urine could } \\
\text { not be } \\
\text { obtained } \\
\text { or patient } \\
\text { refused }\end{array}$ & Negative & Absent & $\begin{array}{l}\text { Irregular } \\
\text { sleep }\end{array}$ \\
\hline 30 & 22 & $\mathrm{Nx}$ & None & $\begin{array}{l}\text { SE: } 94.3 \% \\
\text { SOLmin: } 10.5 \\
\text { REML: } 64.0 \mathrm{~min} \\
\text { AHI: } 5.0 \text { events/h }\end{array}$ & $\begin{array}{l}\text { MSL: } 7.5 \text { min } \\
\text { MREML: } 0 \\
\text { No. naps with } \\
\text { REM: } 0\end{array}$ & Negative & Negative & Absent & $\begin{array}{l}\text { Sleep } \\
\text { deprivation }\end{array}$ \\
\hline 31 & 36 & $\begin{array}{l}\text { Probable } \\
\text { Nx }\end{array}$ & None & $\begin{array}{l}\text { SE: } 62.3 \% \\
\text { SOLmin: } 22.0 \\
\text { REML: } 46.0 \mathrm{~min} \\
\text { AHI: } 10.0 \\
\quad \text { events/h }\end{array}$ & $\begin{array}{l}\text { MSL: } 9.0 \text { min } \\
\text { MREML: } 0 \\
\text { No. naps with } \\
\text { REM: } 0\end{array}$ & $\begin{array}{l}\text { Urine could } \\
\text { not be } \\
\text { obtained } \\
\text { or patient } \\
\text { refused }\end{array}$ & $\begin{array}{l}\text { Blood could } \\
\text { not be } \\
\text { obtained or } \\
\text { patient } \\
\text { refused }\end{array}$ & Present & $\begin{array}{r}\text { Poor sleep } \\
\text { hygiene }\end{array}$ \\
\hline 32 & 60 & $\mathrm{Nx}$ & $\begin{array}{l}\text { Lansoprazole, co-codamol, } \\
\text { domperidone, modafinil }\end{array}$ & $\begin{array}{l}\text { SE: } 64.8 \% \\
\text { SOLmin: } 17.5 \\
\text { REML: } 58.0 \mathrm{~min} \\
\text { AHI: } 24.2 \\
\quad \text { events/h }\end{array}$ & $\begin{array}{l}\text { MSL: } \\
10.4 \text { min } \\
\text { MREML: } 0 \\
\text { No. naps with } \\
\text { REM: } 0\end{array}$ & Positive & Negative & Absent & OSAHS \\
\hline 33 & 20 & $\mathrm{Nx}$ & None & $\begin{array}{l}\text { SE: } 89.6 \% \\
\text { SOLmin: } 15.0 \\
\text { REML: } \\
\quad 147.0 \text { min } \\
\text { AHI: } 7.3 \text { events/h }\end{array}$ & $\begin{array}{l}\text { MSL: } 5.3 \text { min } \\
\text { MREML: } 1.0 \\
\text { No. naps with } \\
\text { REM: } 3\end{array}$ & Negative & Negative & Absent & True Nx \\
\hline 34 & 48 & $\begin{array}{l}\text { Probable } \\
\mathrm{Nx}\end{array}$ & None & $\begin{array}{l}\text { SE: } 56.9 \% \\
\text { SOLmin: } 7.0 \\
\text { REML: } 66.0 \mathrm{~min} \\
\text { AHI: } 4.5 \text { events/h }\end{array}$ & $\begin{array}{l}\text { MSL: } 4.5 \text { min } \\
\text { MREML: } 3.0 \\
\text { No. naps with } \\
\text { REM: } 2\end{array}$ & Negative & $\begin{array}{l}\text { Blood could } \\
\text { not be } \\
\text { obtained or } \\
\text { patient } \\
\text { refused }\end{array}$ & Present & True Nx \\
\hline 35 & 36 & $\mathrm{Nx}$ & None & $\begin{array}{l}\text { SE: } 57.4 \% \\
\text { SOLmin: } 3.0 \\
\text { REML: } 93.5 \mathrm{~min} \\
\text { AHI: } 38.5 \\
\quad \text { events/h }\end{array}$ & $\begin{array}{l}\text { MSL: } \\
11.1 \text { min } \\
\text { MREML: } 0 \\
\text { No. naps with } \\
\text { REM: } 0\end{array}$ & Negative & $\begin{array}{l}\text { Blood could } \\
\text { not be } \\
\text { obtained or } \\
\text { patient } \\
\text { refused }\end{array}$ & Absent & OSAHS \\
\hline 36 & 43 & $\mathrm{Nx}$ & $\begin{array}{l}\text { Salbutamol, beclometasone, } \\
\text { iron tablets }\end{array}$ & $\begin{array}{l}\text { SE: } 68.9 \% \\
\text { SOLmin: } 7.5\end{array}$ & $\begin{array}{l}\text { MSL: } 1.8 \text { min } \\
\text { MREML: } 2.5\end{array}$ & Negative & DQB1*0602 & Present & True Nx \\
\hline
\end{tabular}


Table 1 (continued)

\begin{tabular}{|c|c|c|c|c|c|c|c|c|c|}
\hline Case & Age & $\begin{array}{l}\text { Prior } \\
\text { diagnosis }\end{array}$ & Medication & PSG results & MSLT results & $\begin{array}{l}\text { Drug } \\
\text { screen }^{\mathrm{a}}\end{array}$ & HLA type & $\begin{array}{l}\text { Features } \\
\text { of } \\
\text { cataplexy }\end{array}$ & $\begin{array}{l}\text { Final } \\
\text { diagnosis }\end{array}$ \\
\hline & & & & $\begin{array}{l}\text { REML: } \\
101.0 \mathrm{~min} \\
\text { AHI: } 108.7 \\
\text { events/h }\end{array}$ & $\begin{array}{l}\text { No. naps with } \\
\text { REM: } 1\end{array}$ & & & & \\
\hline 37 & 57 & $\begin{array}{l}\text { Probable } \\
\mathrm{Nx}\end{array}$ & None & $\begin{array}{l}\text { SE: } 70.5 \% \\
\text { SOLmin: } 0.0 \\
\text { REML: } 0 \text { min } \\
\text { AHI: } 18.5 \\
\quad \text { events/h }\end{array}$ & $\begin{array}{l}\text { MSL: } 0.8 \text { min } \\
\text { MREML: } 0.1 \\
\text { No. naps with } \\
\quad \text { REM: } 4\end{array}$ & Negative & DQB1*0602 & Present & True Nx \\
\hline 38 & 34 & $\mathrm{Nx}$ & Diclofenac & $\begin{array}{l}\text { SE: } 77.4 \% \\
\text { SOLmin: } 11.5 \\
\text { REML: } \\
\quad 114.0 \text { min } \\
\text { AHI: } 2.0 \text { events/h }\end{array}$ & $\begin{array}{l}\text { MSL: } 6.9 \text { min } \\
\text { MREML: } \\
10.0 \\
\text { No. naps with } \\
\text { REM: } 1\end{array}$ & Negative & $\begin{array}{l}\text { DRB1*15, } \\
\text { DRB5 } * 01 / \\
02, \\
\text { DQB } 1 * 06, \\
\text { DQA }\end{array}$ & Absent & $\begin{array}{r}\text { Poor sleep } \\
\text { hygiene }\end{array}$ \\
\hline 39 & 49 & $\mathrm{Nx}$ & $\begin{array}{l}\text { Olanzapine, aspirin, metformin, } \\
\text { atorvastatin, furosemide, } \\
\text { diclofenac, co-codamol, } \\
\text { levothyroxine, } \\
\text { hydrocortisone, }\end{array}$ & $\begin{array}{l}\text { SE: } 78.8 \% \\
\text { SOLmin: } 40.5 \\
\text { REML: } \\
107.0 \mathrm{~min} \\
\text { AHI: } 16.5 \\
\quad \text { events } / \mathrm{h}\end{array}$ & $\begin{array}{l}\text { MSL: } \\
15.5 \text { min } \\
\text { MREML: } 0 \\
\text { No. naps with } \\
\text { REM: } 0\end{array}$ & Negative & Negative & Absent & OSAHS \\
\hline 40 & 62 & $\mathrm{Nx}$ & $\begin{array}{l}\text { Fentanyl patch, levothyroxine, } \\
\text { bendroflumethia-zide, aspirin, } \\
\text { amitriptyline, acetaminophen, } \\
\text { diazepam, perindopril }\end{array}$ & $\begin{array}{l}\text { SE: } 29.8 \% \\
\text { SOLmin: } 62.0 \\
\text { REML: } 0 \text { min } \\
\text { AHI: } 7.2 \text { events/h }\end{array}$ & $\begin{array}{l}\text { MSL: } \\
20.0 \text { min } \\
\text { MREML: } 0 \\
\text { No. naps with } \\
\text { REM: } 0\end{array}$ & Negative & Negative & Absent & OSAHS \\
\hline 41 & 63 & $\mathrm{Nx}$ & $\begin{array}{l}\text { Amitryptiline, gliclazide, } \\
\text { metformin, simvastatin }\end{array}$ & $\begin{array}{l}\text { SE: } 37.9 \% \\
\text { SOLmin: } 6.5 \\
\text { REML: } 97.0 \mathrm{~min} \\
\text { AHI: } 71.1 \\
\quad \text { events/h }\end{array}$ & $\begin{array}{l}\text { MSL: } 8.4 \text { min } \\
\text { MREML: } \\
0.8 \text { No. } \\
\text { naps with } \\
\text { REM: } 2\end{array}$ & Negative & Negative & Present & True Nx \\
\hline
\end{tabular}

Abbreviations: $P S G$ polysomnography, MSLT multiple sleep latency test, $H L A$ human leukocyte antigen, $N x$ narcolepsy, $S E$ sleep efficiency, $R E M L$ rapid eye movement latency, $A H I$ apnea hypogea index, $M S L$ mean sleep latency, $R E M$ rapid eye movement, $O S A H S$ obstructive sleep apnea hypoventilation syndrome, $S O L$ sleep onset latency, MREML mean rapid eye movement latency

${ }^{a}$ We routinely screen for drugs of abuse consisting of opiates, amphetamines, cocaine, methadone and benzodiazepines. Cannabinoids are also included

Fig. 1 Drug treatment at referral for 41 patients referred with a 'diagnosis of narcolepsy'. SSRI selective serotonin re-uptake inhibitor, SNRI serotoninnorepinephrine re-uptake inhibitor, TCA tricyclic antidepressant

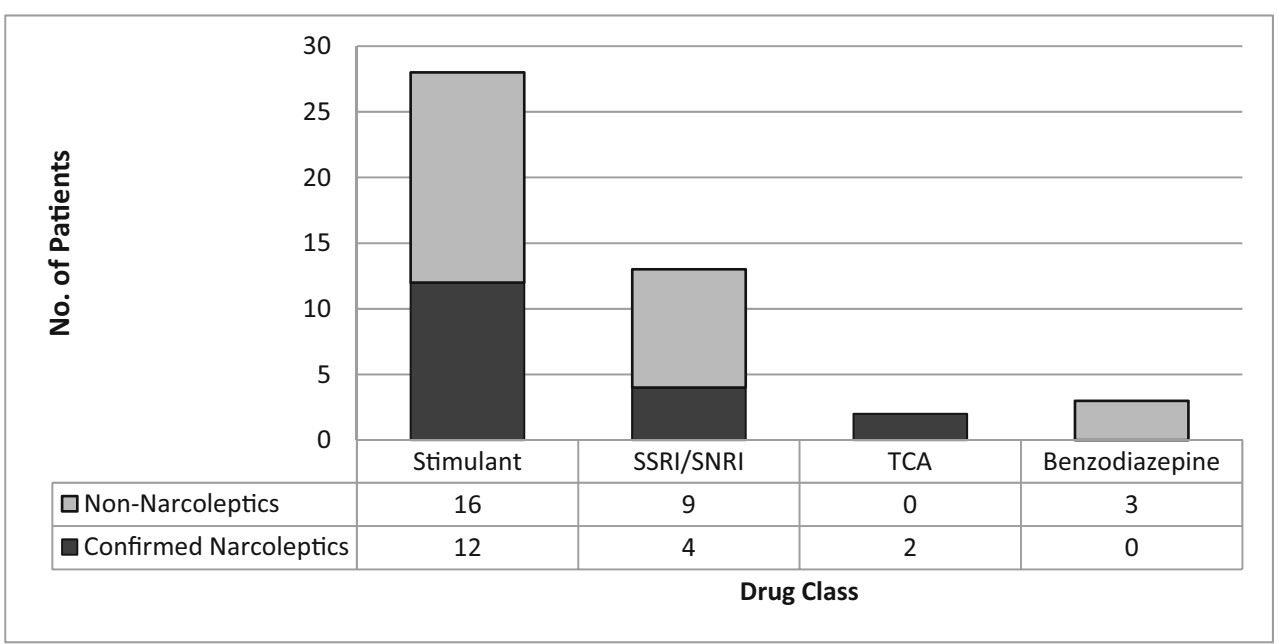


Prior to referral, 31 patients were prescribed CNS-altering medication (Fig. 1). Of 28 patients receiving stimulants, 16 did not have narcolepsy. The specific drugs used in this latter patient group were modafinil $(n=10)$, dexamphetamine $(n=5)$ and methylphenidate $(n=1)$.

\section{Discussion}

A diagnosis of narcolepsy was incorrectly attributed to almost $50 \%$ of patients referred for further opinion by a variety of specialists and generalists. We are one of the only specialized sleep clinics in the country; therefore, the high number of innacurately diagnosed patients could result from referral bias. Easily recognized and accurately diagnosed cases of narcolepsy which do not require clarification would possibly not be referred to the sleep clinic for validation and we may be receiving only cases that pose difficult diagnostic conundrums for referring doctors. The only significant difference between narcoleptics and non-narcoleptics on presentation was a history of cataplexy, but this was not exclusive to narcoleptics. [6] Objective testing using PSG and MSLT was discriminatory between groups, in keeping with previous studies. [7].

Accurate diagnosis of narcolepsy is critical for many reasons, such as the impact it has on quality of life, driving, employment, insurance and implications regarding pregnancy in women. Treatment involves the use of controlled (e.g. amphetamines) and expensive (e.g. sodium oxybate) drugs which should not be prescribed in error, as there are not inconsiderable potential side effects (e.g. nausea, weight loss, psychiatric complications), tolerance and possible addiction issues. [1] Health service costs resulting from inappropriate treatment are also an important consideration. [1, 3, 6] Furthermore, several countries require a diagnosis of a medical condition in order to approve prescription medication. With the use of the Internet and increasing information disseminated on television programmes, many people self-diagnose, presenting at clinics with highly educated information and an excellent history of the disorder. This can be problematic because drugseeking individuals may present with fabricated narcolepsy symptoms in order to obtain stimulant medications. [14] The use of illegal substances can also influence the results of MSLT and urine drug screening remains of great importance in accurate diagnosis and treatment of adults and children. $[2,16]$.

The study sample of 41 patients over a 6 -year time period-with 19 patients correctly diagnosed with narcolepsymight be considered small. However, the figures are high when taken in the context of narcolepsy being an uncommon disorder with a prevalence of $0.01 \%$ in the general population. A limitation to our study is that we were unable to calculate the degree of certainty of diagnosis and if the source of initial diagnosis had an impact on the confirmation rate due to the small sample size. In an age when access to specialized diagnostic facilities is unrestricted, it is of concern that such diagnostic inaccuracy continues to occur. It is hoped that the recent publication of the ICSD-3 with its detailed instructions on diagnosing narcolepsy will further reduce misdiagnosis by giving a clearer and more accurate description for providers to use.

\section{Compliance with ethical standards}

Funding No funding was received for this research.

Conflict of interest All authors certify that they have no affiliations or involvement with any organization or entity with any financial interest (such as honoraria; educational grants; participation in speakers' bureaus; membership, employment, consultancies, stock ownership or other equity interest; and exert testimony or patent-licensing arrangements) or nonfinancial interest (such as personal or professional relationships, affiliations, knowledge or beliefs) in the subject matter or materials discussed in this manuscript.

Ethical approval All procedures performed in studies involving human participants were in accordance with the ethical standards of the institutional and/or national research committee and with the 1964 Helsinki declaration and its later amendments or comparable ethical standards.

Informed consent This study does not have any informed consent.

Authors' contributions Laura Dunne did the writing, analysis and primary data collection. Pallavi Patel and Emily L Maschauer did the writing and editing. Ian Morrison did the writing, editing and design. Renata Riha did the design, analysis, writing and editing.

Disclosures Laura Dunne, Pallavi Patel, Emily L Maschauer, Ian Morrison and Renata Riha report no disclosures with respect to this study.

Open Access This article is distributed under the terms of the Creative Commons Attribution 4.0 International License (http:// creativecommons.org/licenses/by/4.0/), which permits unrestricted use, distribution, and reproduction in any medium, provided you give appropriate credit to the original author(s) and the source, provide a link to the Creative Commons license, and indicate if changes were made.

\section{References}

1. Mignot EJ (2012) A practical guide to the therapy of narcolepsy and hypersomnias syndromes. Neurotherapeutics 9(4):739-752

2. Morrison I, Riha RL (2012) Excessive daytime sleepiness and narcolepsy-an approach to investigation and management. European Journal of Internal Medicine 23(2):110-117

3. Morrison I, Buskova J, Nevšimalová S, Douglas NJ, Riha RL (2011) Diagnosing narcolepsy with cataplexy on history alone: challenging the international classification of sleep disorders (ICSD-2) criteria. Eur J Neurol 18(7):1017-1020

4. Broughton WA, Broughton RJ (1994) Psychosocial impact of narcolepsy. Journal of Sleep 17(8):S45-S49

5. Johns MW (1991) A new method for measuring daytime sleepiness: the Epworth sleepiness scale. Journal of Sleep 14(6):540-545

6. Krahn LE, Gonzalez-Arriaza HL (2004) Narcolepsy with cataplexy. Am J Psychiatr 161(12):2181-2184 
7. Littner MR, Kushida C, Wise M, Davila DG, Morgenthaler T, LeeChiong T, et al. (2005) Practice parameters for clinical use of the multiple sleep latency test and the maintenance of wakefulness test. Journal of Sleep 28(1):113-121

8. Benca RM (2008) Sleep in psychiatric disorders. In: ACCP sleep medicine board review course. Northbrook, Illinois, pp. 71-87

9. American Academy of Sleep Medicine (2005) International classification of sleep disorders - second edition (ICSD-2). American Academy of Sleep Medicine, Westchester

10. American Academy of Sleep Medicine (2014) International classification of sleep disorders - third edition (ICSD-3). American Academy of Sleep Medicine, Westchester

11. Silber MH, Krahn LE, Olson EJ (2002) Diagnosing narcolepsy: validity and reliability of new diagnostic criteria. Sleep Med 3(2): 109-113
12. Trotti LM, Staab BA, Rye DB (2013) Test-retest reliability of the multiple sleep latency test in narcolepsy without cataplexy and idiopathic hypersomnia. J Clin Sleep Med 9(8):789-795

13. Dauvilliers Y, Arnulf I, Mignot E (2007) Narcolepsy with cataplexy. Lancet 10(369):499-511

14. Wilford BB (1990) Abuse of prescription drugs. West J Med 152(5):609-612

15. Arand D, Bonnet M, Hurwitz T, Mitler M, Rosa R, Sangal RB (2005) The clinical use of the MSLT and MWT. Sleep 28:123-144

16. Dzodzomenyo S, Stolfi A, Splaingard D, Earley E, Onadeko O, Splaingard M (2015) Urine toxicology screen in multiple sleep latency test: the correlation of positive tetrahydrocannabinol, drug negative patients, and narcolepsy. J Clin Sleep Med 11(2):93-99 